University of Nebraska - Lincoln

DigitalCommons@University of Nebraska - Lincoln

Response of Tribolium castaneum and Tribolium confusum adults to vertical black shapes and its potential to improve trap capture

\author{
Altair A. Semeao \\ Kansas State University \\ James F. Campbell \\ USDA, james.campbell@usda.gov \\ R. Jeff Whitworth \\ Kansas State University \\ Phillip E. Sloderbeck \\ Kansas State University
}

Follow this and additional works at: https://digitalcommons.unl.edu/usdaarsfacpub

Part of the Agricultural Science Commons

Semeao, Altair A.; Campbell, James F.; Whitworth, R. Jeff; and Sloderbeck, Phillip E., "Response of Tribolium castaneum and Tribolium confusum adults to vertical black shapes and its potential to improve trap capture" (2011). Publications from USDA-ARS / UNL Faculty. 840.

https://digitalcommons.unl.edu/usdaarsfacpub/840

This Article is brought to you for free and open access by the U.S. Department of Agriculture: Agricultural Research Service, Lincoln, Nebraska at DigitalCommons@University of Nebraska - Lincoln. It has been accepted for inclusion in Publications from USDA-ARS / UNL Faculty by an authorized administrator of DigitalCommons@University of Nebraska - Lincoln. 


\title{
Response of Tribolium castaneum and Tribolium confusum adults to vertical black shapes and its potential to improve trap capture
}

\author{
Altair A. Semeao a , James F. Campbell ${ }^{\text {b, }}{ }^{*}$, R. Jeff Whitworth ${ }^{\text {a }}$, Phillip E. Sloderbeck ${ }^{\mathrm{c}}$ \\ ${ }^{a}$ Department of Entomology, Kansas State University, Manhattan, KS 66506, United States \\ ${ }^{\mathrm{b}}$ USDA, ARS, Center for Grain and Animal Health Research, 1515 College Ave., Manhattan, KS 66502, United States \\ ${ }^{\mathrm{c}} \mathrm{KSU}$-Southwest Research and Extension Center, 4500 E. Mary St., Garden City, KS 67846, United States
}

\section{A R T I C L E I N F O}

\section{Article history:}

Accepted 12 January 2011

\section{Keywords:}

Color

Red flour beetle

Confused flour beetle

Behavior

Pheromone trapping

\begin{abstract}
A B S T R A C T
Tribolium castaneum and Tribolium confusum can be monitored in food processing facilities using traps baited with pheromones and kairomones, but beetle response to traps might be enhanced by adding visual cues. Against a white background, T. castaneum adults were more likely to visit black pillars than white pillars when presented with a choice (e.g., $73 \%$ of beetles visited black and $17 \%$ visiting white pillar), and visits to black pillars increased with pillar height. When tested against a black background, beetles did not show a significant preference for either color pillar regardless of height. When comparing beetle's captures in pheromone/kairomone baited traps placed in front of a white or black panel in a white arena under high, low, or dark light conditions, more beetles were captured in traps in front of black panels under both high and low light conditions, but not under dark conditions. A similar pattern of capture under low light and dark conditions was also found for the closely related species T. confusum. In a larger scale choice test, the same pattern of greater T. castaneum captures in traps in front of black panels than white panels was obtained, whether traps were placed in corners or along walls. Our results suggest that captures in monitoring traps could be increased by adding dark vertical shapes behind trap locations or placing traps near dark structures.
\end{abstract}

Published by Elsevier Ltd.

\section{Introduction}

Integrated pest management of stored-product insects in food processing facilities relies on monitoring data to guide management decisions and to evaluate the effectiveness of the program (Barak et al., 1990; Burkholder, 1990). Often this monitoring data is generated using pheromone and/or kairomone baited traps that rely on insect olfaction to facilitate captures, but typically don't exploit other sensory modalities such as vision (Chambers, 1990). However, in other systems color preferences by economically important insects have been demonstrated (Southwood, 1978; Prokopy and Owens, 1983), and visual cues, typically attractive colors and trap shapes, have been exploited to monitor and manage insect pests (e.g., Hoback et al., 1999; Strom and Goyer, 2001; Athanassiou et al., 2004). Although response to color and shape by stored-product insects is relatively poorly understood, traps that combine olfactory cues such as pheromones and kairomones with visual cues may offer the potential to increase captures of storedproduct insects.

\footnotetext{
* Corresponding author. Tel.: +1 785776 2717; fax: +1 7855375584 .

E-mail address: james.campbell@ars.usda.gov (J.F. Campbell).
}

Exploitation of visual signals in insect monitoring programs fall into three general categories: lights that attract insects, colored objects that are attractive because of their specific reflectance, and shapes or silhouettes that stand out against a contrasting background. Pinero et al. (2006) found that female melon flies (Bactrocera cucurbitae (Coquillett)) were primarily attracted to objects of hemispherical shape associated with either yellow, white, or orange pigments. Mainali and Lim (2010) found that western flower thrips (Frankliniella occidentalis (Pergande)) were more attracted to flat yellow sticky traps on a black background and suggested that the black background may help the insect to perceive reflectance with minimal interception from other sources of reflectance. There is considerable variation among insect species in their color preference, but attractive colors typically resemble suitable habitats for mating, oviposition, or feeding (e.g., Hoback et al., 1999; Cilek, 2003; Döring et al., 2004; López-Guillén et al., 2009). For example, many herbivorous insects respond to the color yellow or white, which corresponds to the peak color reflectance of plants (Prokopy and Owens, 1983). Insect species that attack trees such as bark beetles respond to vertical trunk silhouettes for landing and short range orientation, and traps that provide appropriate visual silhouettes are usually more efficient (Lanier, 1983; Finch and Collier, 2000). 
Tribolium castaneum (Herbst), red flour beetle, and Tribolium confusum Jacquelin du Val, confused flour beetle, (Coleoptera: Tenebrionidae) are major pests of food processing facilities, especially wheat and rice mills, and walking beetles can be monitored using traps based on a pitfall design (e.g., Mullen, 1992) and baited with pheromone and kairomone attractants. These traps have been used successfully to monitor pest populations of $T$. castaneum (e.g., Campbell et al., 2010), but the level of attraction by flour beetles to these baited traps is widely, although anecdotally, reported as relatively weak. Reza and Parween (2006) showed that T. castaneum larvae and adults differed in their tendency to aggregate on different colors when presented with a choice between different color surfaces. Of the colors tested in this study, adults of T. castaneum exhibited a tendency to aggregate only on black surfaces. Here we evaluate if adult $T$. castaneum are actually attracted to the color black and if the height of the black shape impacts the level of attraction. We also evaluate if these black shapes when combined with a commercially available pheromone/kairomone baited trap can increase the effectiveness of these traps at capturing beetles. Finally, we confirmed if $T$. confusum exhibited a similar increase in trap captures with black shapes.

\section{Materials and methods}

\subsection{Experimental conditions}

Tribolium castaneum and T. confusum adults used in the experiments were obtained from laboratory colonies maintained in 0.941 glass jars containing wheat flour fortified with $5 \%$ (by weight) brewer's yeast (ICN Biomedicals, Aurora, $\mathrm{OH}$ ). Both colonies were established within 10 months before experiments were conducted by mixing $\sim 100$ adults collected from food facilities in Kansas, USA The colonies were maintained at $28.0 \pm 0.5{ }^{\circ} \mathrm{C}, 60.0 \pm 5.0 \%$ r.h. and 14:10 light:dark cycle (mean \pm SE). Only adults less than two months old were used in experiments and collected the same day as experiments were conducted. Beetles were held for at least $30 \mathrm{~min}$ in Petri dishes without food inside the experimental chambers prior to the start of the experiment. Individual beetles were only used once in an experiment.

Experiment 1 was conducted inside a chamber $(4.7 \mathrm{~m}$ long by $4.7 \mathrm{~m}$ wide by $2.4 \mathrm{~m}$ tall) located in a room without controlled temperature and r.h. and where lighting resulted primarily from natural sunlight. To reduce environmental variability all replicates were performed between $1300 \mathrm{~h}$ and $1600 \mathrm{~h}$. The mean \pm SE environmental conditions in chamber during experiments were $21.3 \pm 0.4{ }^{\circ} \mathrm{C}$ air temperature, $22.7 \pm 0.8 \%$ r.h., and $380.5 \pm 2.3$ lux light intensity. All subsequent experiments were conducted in an environmental chamber ( $6.1 \mathrm{~m}$ long by $4.9 \mathrm{~m}$ wide by $2.4 \mathrm{~m}$ tall) held at $25.0 \pm 0.0^{\circ} \mathrm{C}, 66.5 \pm 0.0 \%$ r.h. and $24 \mathrm{~h}$ light $(214.9 \pm 0.3$ lux $)$. Temperature and r.h. were measured using either a data logger $\left(\mathrm{HOBO}^{\circledR} \mathrm{H} 8\right.$ family, Onset Computer Corp., Pocasset, MA) or handheld meter (Kestrel ${ }^{\circledR} 3000$, Nielsen-Kellerman, Boothwyn, PA), and light intensity was monitored using light meter (Model EA33, Extech Instruments Corporation, Waltham, MA).

\subsection{Experiment 1: are T. castaneum adults attracted to black shapes?}

Beetle response when presented with a choice between black or white pillars of different heights, when the background color was either black or white, was evaluated. This enables us to determine if beetle response was due to the dark shape or just due to contrast with the background. An experimental arena was constructed inside chamber described above from four foam core boards $(1 \times 1 \mathrm{~m}$ squares) taped together to form a cube, with the top open and the floor of the chamber forming the floor of the box. Depending on the treatment conditions, the inside of the box was covered with either white or black paper (Art Kraft Duo-Finish paper, C2F Inc., Beaverton, OR). We created three different types of cuboids (pillars) out of corrugated cardboard with dimensions of $14 \times 14 \mathrm{~cm}$ and height of either: $2 \mathrm{~cm}$ (short), $14 \mathrm{~cm}$ (medium), or $33 \mathrm{~cm}$ (tall). The heights of the pillars were selected to give a range of heights, with the tallest pillar height predicted to be perceived by the beetle as an extremely tall shape given the size of the beetle and the release distance used in the first experiment. The pillars were covered with the same type of paper used inside the box, either black or white depending on the treatment conditions.

At the center of the arena floor, a square $(50 \times 50 \mathrm{~cm})$ was marked off with a pencil and this was the observation zone for recording beetle behavior. In each replicate, we placed one black pillar and one white pillar of the same height at opposite sides of the square marked on the floor (adjacent to the line but outside the observation zone). Individual $T$. castaneum adults were placed inside $1.5 \mathrm{~mL}$ centrifuge tubes, acclimated under conditions in arena prior to release, and then released by placing an opened and inverted tube at the center of the observation zone and removing the tube after beetle inside was observed to upright and walking. After release, the time it took for either of the following events to occur was recorded: reach the edge of the observation zone, reach the black pillar, or reach the white pillar. Observations were terminated after either of these three events occurred or if $300 \mathrm{~s}$ had elapsed. Replications consisted of individual beetles exposed to pairs of pillars of the same height against either a black or a white background. Within, first the white and then the black arena color, the order of the pillar height pairs was completely randomized, and within each height the orientation of the pillars was alternated (North-South and East-West orientations). A total of 25 replicates of each treatment were performed against the black background, and a total of 30 replicates of each treatment, except for one treatment that had 31 replicates, were performed against the white background.

Data was analyzed in SAS version 9.1.2 (SAS Institute, Cary NC) using contingency table analysis (PROC FREQ). For each color background, the contingency tables had 3 rows (pillar height) and three columns (beetles reach edge, reach black pillar, reach white pillar). From a total of 166 beetles tested, only 3 beetles remained in the observation zone for the $300 \mathrm{~s}$ and they were excluded from analysis. Chi-square test was used for testing the null hypothesis that the size of the pillars did not affect the events and to evaluate differences in the number of beetles reaching black and white pillars.

\subsection{Experiment 2: how is the movement behavior of T. castaneum impacted by black pillars of different heights?}

Movement pathways of individual beetles when exposed to pillars of different colors and heights were evaluated in a no-choice experiment. Individual beetles were observed in a wood arena (120 cm long by $120 \mathrm{~cm}$ wide and $23.5 \mathrm{~cm}$ tall) painted white (Zinsser Bulls Eye 1-2-3 primer, Rustoleum, Vernon Hills, IL) and held in chamber and under environmental conditions described above. The wood arena was covered by a box made of white paper (Art Kraft Duo-Finish paper) $(120 \mathrm{~cm}$ long by $120 \mathrm{~cm}$ wide by $130 \mathrm{~cm}$ tall) with an observation hole cut in the center of the top through which a video camera could be inserted. This paper box enabled a video camera to be placed at the necessary height above the floor of the arena to record beetles and also provided a monochromatic white environment in which to observe response to black shape. The observation zone inside the wood arena in which activity of individual beetles was recorded was $50 \times 50 \mathrm{~cm}$. 
Beetle behavior in each replicate was recorded over a period of $180 \mathrm{~s}$ using a portable digital video camera recorder (Model HDRXR520V, Sony Electronics Inc., San Diego, CA) placed $130 \mathrm{~cm}$ above the floor of the arena. Digital video files were transferred to a computer and beetle positions determined and movement pathways analyzed using EthoVision 3.0 (Noldus Information Technology, Wageningen, The Netherlands). The following pathway metrics were calculated and analyzed: heading (degrees), distance moved $(\mathrm{cm})$, velocity $(\mathrm{cm} / \mathrm{s})$, turn angle (degrees), angular velocity (degrees/s) and meander (degrees/cm). The time it took to reach the pillar or the edge of the observation zone was measured.

In each replication, we placed one pillar (short white, short black, tall white, tall black as described in experiment 1) along the edge of the observation zone, and a total of twenty two beetles were tested in each treatment. The order of the four pillar heights was completely randomized and the orientation of the pillars within a given height was rotated in a clockwise direction (at least 5 replications in each direction). Between replicates the arena was sprayed with water and cleaned with paper towel.

Survival or time-to-event analysis followed by pairwise multiple comparison procedures (Holm-Sidak method) in SigmaPlot (SigmaPlot version 11, Systat Software, Chicago, IL), and general linear models (PROC GLM) procedure and contingency table analysis in SAS were used to evaluate differences among treatments (pillar height and color). Contingency table analysis was performed using a four column (pillar height) and two row (visit or not visit pillar) table. Additionally, heading (direction of movement relative to a reference line) data was analyzed for deviation from uniform distribution using circular statistics and mean heading compared among treatments using Watson-Williams test (Zar, 1999). Because the location of the pillar rotated among replications, to facilitate comparisons heading values calculated by EthoVision software were transposed so that all headings were relative to a reference line from release point to pillar. Relative heading angles $\left(0^{\circ}-180^{\circ}\right)$ were used rather than absolute heading angles $\left(0^{\circ}-360^{\circ}\right)$.

\subsection{Experiment 3: does the use of black shape increase Tribolium spp. captures in pheromone and kairomone baited traps in simple landscape?}

To determine if increased attraction to black shapes could increase captures in traps we measured the number of beetles captured in pheromone/kairomone baited traps when placed against black or white panels under different light intensity conditions. The pheromone trap used was the Dome trap baited with pheromone lure for Tribolium spp. and 15 drops of food oil (Trécé Inc., Adair, OK, USA). Two traps were used in each replicate, with traps placed against two opposite interior walls of the arena described in Experiment \#2. Behind each trap we placed a piece of $0.03 \mathrm{~mm}$ galvanized metal $(7.5 \mathrm{~cm}$ wide by $18 \mathrm{~cm}$ tall) painted either flat black or flat white (ColorPlace ${ }^{\circledR}$ - Fast dry spray paint, Wal-Mart Stores, Inc., Bentonville, AR). Three light intensities - high, low and dark - were tested and these corresponded to $2160.0 \pm 12.6,98.1 \pm 0.6$, and $0.0 \pm 0.0$ lux, respectively. Light intensity was measured at arena floor level at the midpoint of each wall. Different light intensities were obtained by placing four $120 \mathrm{~cm}$ long fluorescent light bulbs $80 \mathrm{~cm}$ above the arena and covering the arena with a sheet of translucent plastic. For dark treatment all lights in the environmental chamber were turned off, for the high light intensity treatment lights in the chamber were turned on and arena only covered with the plastic sheet, but for low light conditions three layers of paper (2 layers of white color and 1 layer of brown color Kraft paper) were placed on the top of the plastic. Temperature in the chamber and inside the arena at different light intensities was measured. The temperature in the chamber when the lights were turned on was $25.1{ }^{\circ} \mathrm{C} \pm 0.0{ }^{\circ} \mathrm{C}$, and when the lights were turned off was $24.9{ }^{\circ} \mathrm{C} \pm 0.0{ }^{\circ} \mathrm{C}$. The temperature inside the arena when the lights in the chamber were turned off but the four fluorescent light bulbs were turned on in the high light intensity treatment was $25.4{ }^{\circ} \mathrm{C} \pm 0.0{ }^{\circ} \mathrm{C}$.

At the beginning of each replicate, $T$. castaneum adults were released by placing a plastic Petri dish ( $9 \mathrm{~cm}$ diameter) containing 100 unsexed beetles at the center of the arena and using four pieces of folded paper $(1.2 \mathrm{~cm}$ wide by $7.5 \mathrm{~cm}$ long) to create ramps that beetles could use to leave the dish. Twenty-four hours after release, the number of beetles in each trap and in the arena was counted. Between replicates all beetles were removed, arena cleaned with $70 \%$ ethanol, and left uncovered for about $30 \mathrm{~min}$. A new set of Dome traps and pheromone lures (aerated for $24 \mathrm{~h}$ prior to start of experiment) was used for each replication. Eight replications (at each light intensity) were performed and the order of the treatments was completely randomized. Within a light intensity and between replications, the walls that the traps and metal plates were placed against were rotated.

In a similar experimental protocol, we investigated if the closely related species $T$. confusum would have similar responses to the traps with different color panels. The only difference in the design for this species was that based on the results with T. castaneum, we excluded the high light intensity treatment.

To determine if the number captured differed between black and white panels, pairwise comparisons between the two colors at each light intensity, were performed using $t$-tests or the nonparametric Mann-Whitney Rank Sum tests using SigmaPlot software. Nonparametric test was used when data did not always fit a normal distribution or pass the equal variance test. For comparisons among traps with same panel color at the different light intensities, we applied the general linear models (GLM) procedure and Tukey mean comparison test $(a l p h a=0.05)$ using SAS software. Treatment means and SEMs are presented in the text and figures.

\subsection{Experiment 4: does the use of black shape increase T. castaneum captures in pheromone and kairomone baited traps in a more complex landscape?}

Impact of black shapes behind traps on beetle captures in a larger and more complex landscape was investigated using the complete extent $\left(29.7 \mathrm{~m}^{2}\right)$ of the environmental chamber with a table ( $86 \mathrm{~cm}$ wide by $152 \mathrm{~cm}$ long by $78 \mathrm{~cm}$ tall) and wind tunnel (86 $\mathrm{cm}$ wide by $223 \mathrm{~cm}$ long by $169 \mathrm{~cm}$ tall) placed next to each other in center of chamber. The chamber interior walls were white and experiments were run with lights on $24 \mathrm{~h}(484.6 \pm 50.5$ lux light intensity at floor level in corners and midpoints of walls). In the first set of experiments (four replicates), four Dome traps with pheromone lures and food oil, as described above, were placed in each corner of the chamber. In the second set of experiments (four replicates), Dome traps were placed at the midpoint along each of the walls. Pieces of $18 \mathrm{~cm}$ wide by $100 \mathrm{~cm}$ tall $(0.5 \mathrm{~mm}$ thick) foam board covered with either black or white paper were placed behind each trap. Two opposite traps had black backgrounds and two opposite traps had white backgrounds, and their positions were rotated between replicates.

For each replicate, $300 \mathrm{~T}$. castaneum adults were released using a Petri dish with ramps, as described above, placed on floor in the center of the chamber. Twenty-four hours after release, number of beetles in each trap and remaining in the chamber were counted. For analysis, number of beetles in traps with the same color shape were combined and total number captured compared using paired $t$-tests (SigmaPlot). Traps placed in corners or along walls were analyzed separately. Between replicates all visible beetles were 
removed and chamber cleaned using an insect vacuum (Model 2820GA, BioQuip Products, Rancho Dominguez, CA, USA) and a broom.

\section{Results}

\subsection{Experiment 1: are T. castaneum adults attracted to black shapes?}

In the arena with a white background, the number of beetles visiting a pillar was significantly affected by pillar color and height ( $3 \times 3$ contingency table analysis, $\chi^{2}=11.8, \mathrm{df}=4, P=0.019$ ). When given a choice between short pillars, the number of beetles visiting each pillar color was not statistically different $(2 \times 2$ contingency table analysis, $\chi^{2}=1.35, \mathrm{df}=1, P=0.246$ ): $35.7 \%$ of the beetles visited the black and $14.3 \%$ visited the white pillar. When given a choice between medium pillars, significantly more beetles visited black pillars $(58.1 \%)$ compared to white pillars $(12.9 \%)(2 \times 2$ contingency table analysis, $\left.\chi^{2}=4.96, \mathrm{df}=1, P=0.026\right)$. When given a choice between tall pillars, significantly more beetles visited black pillars (73.3\%) than visited white pillars $(16.7 \%)(2 \times 2$ contingency table analysis, $\left.\chi^{2}=5.94, \mathrm{df}=1, P=0.015\right)$. However, when choice tests were performed in arenas with black background, the height and color of the pillars did not significantly affect the number of beetles visiting the two pillar colors $(3 \times 3$ contingency table analysis, $\left.\chi^{2}=1.98, \mathrm{df}=4, P=0.74\right)$.

\subsection{Experiment 2: how is the movement behavior of T. castaneum impacted by black pillars of different heights?}

The number of beetles visiting the pillars of different height and color was statistically different among the four treatments $(4 \times 2$ contingency table analysis, $\left.\chi^{2}=15.19, \mathrm{df}=3, P=0.002\right)$ : 0 out of 15 $(0.0 \%)$ visited the short white pillar, 1 out of $15(6.7 \%)$ visited the tall white, 5 out of 21 (23.8\%) visited the short black pillar, and 10 out of $20(50.0 \%)$ visited the tall black pillar. Some of the beetles released tried to fly during the observation period, and these individuals were excluded from analysis. Interestingly, more beetles tended to fly in the white pillar treatments compared to the black pillar treatments: 7 beetles with short white pillar, 7 beetles with tall white pillar, 1 beetle with short black pillar, and 2 beetles with tall black pillars, out of a total of 22 beetles released in each treatment.

The only calculated movement parameters that showed statistical differences among treatments $(P<0.05)$ were time spent in the arena and heading. Using survival analysis to evaluate time spent in observation zone, there were significant differences among treatments (survival analysis log-rank test, $\chi^{2}=8.92$, $\mathrm{df}=3$, $P=0.03$ ) (Fig. 1). Comparing individual treatments, time spent in observation zone was only significantly different between short white and tall black pillar treatments (log-rank test with HolmSidak method, $\chi^{2}=8.74, \mathrm{df}=1, P=0.003$ ); beetles reached the edge more quickly when the tall black pillar was present than when the short white pillar was present.

When evaluating the heading of released beetles, only the tall black pillar treatment had a circular distribution that was significantly different from uniform (circular analysis, $z=3.019$, $\mathrm{df}=20$, $P<0.05$ ). Comparison of mean heading across the four treatments showed significant differences among treatments (Williams Watson test, $F=8.34, \mathrm{df}=3, P<0.05$ ) (Fig. 2A). Pairwise comparisons showed that mean heading of beetles in black pillar treatments were statistically lower than beetles in white pillar treatments, indicating a more directed movement toward pillar, but heading did not differ among pillar heights within a pillar color (Fig. 2A). Pairwise comparisons performed with Williams Watson test showed differences between: tall black and tall white $(F=10.56$,

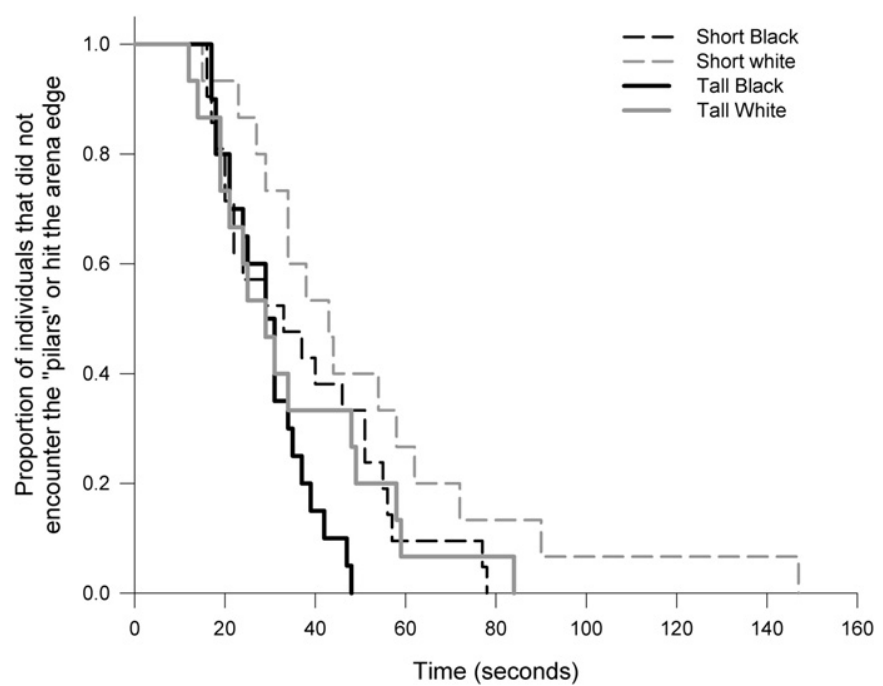

Fig. 1. Time-to-event (survival) curves for the time after individual T. castaneum adults are released until they reach the edge of the observation zone, including encountering the pillars aligned with the edge, when exposed to black or white pillars of different heights.

df $=1, P<0.05)$; tall black and short white $(F=11.38, \mathrm{df}=1$, $P<0.05)$; short black and short white $(F=6.77, \mathrm{df}=1, P<0.05)$; short black and tall white $(F=10.29, \mathrm{df}=1, P<0.05)$.

Distance moved (GLM, $F=1.95, \mathrm{df}=3, P=0.131$ ) (Fig. 2B), velocity (GLM, $F=0.15, \mathrm{df}=3, P=0.927$ ) (Fig. $2 \mathrm{C}$ ), turn angle (GLM, $F=0.73, \mathrm{df}=3, P=0.537$ ) (Fig. $2 \mathrm{D})$, angular velocity (GLM, $F=0.44$, $\mathrm{df}=3, P=0.727$ ) (Fig. 2E) and meander (GLM, $F=0.35, \mathrm{df}=3$, $P=0.787$ ) (Fig. 2F) were not significantly different among treatments. There was an interesting trend for movement path metrics to have less variance in the tall black pillar treatment compared to treatments with white pillars (Fig. 2).

\subsection{Experiment 3: does the use of black shape increase Tribolium spp. captures in pheromone and kairomone baited traps in simple landscape?}

Pairwise comparisons of $T$. castaneum captures between traps with black and white shapes, showed that under high and low light intensity conditions traps with black shapes had greater captures than traps with white shapes (high light intensity: paired $t$-test, $t=2.61, \mathrm{df}=14, P=0.035$; low light intensity: Mann-Whitney test, $U=9.00, \mathrm{df}=14, P=0.015)$. Under dark conditions, there was no difference in captures between traps with white or black shapes (paired $t$-test, $t=-0.82, \mathrm{df}=14, P=0.44$ ) (Fig. 3A).

The total number of $T$. castaneum captured in both traps under low light intensity $(77.9 \pm 4.6)$ was greater than the number captured under dark conditions $(49.3 \pm 8.5)$, but total number captured in the high light intensity treatment $(65.4 \pm 8.8)$ did not differ from either of the other treatments (GLM, $F=3.61$, $\mathrm{df}=2$, $P=0.045$ ). Comparing beetle captures just in traps with black shapes among the different light intensities revealed that more beetles were captured under the low light intensity $(45.8 \pm 5.2)$ compared to dark $(23.1 \pm 5.0)$ treatments, but number captured under high light intensity $(40.6 \pm 6.0)$ did not differ from other light intensities (GLM, $F=4.83, \mathrm{df}=2, P=0.019$ ) (Fig. 3B). No differences were detected in beetle captures in traps with white shapes among the different light intensities (GLM, $F=0.95, \mathrm{df}=2, P=0.402$ ) (Fig. 3C).

A similar pattern of black shapes increasing trap captures was observed for T. confusum, with beetle captures in traps with black 

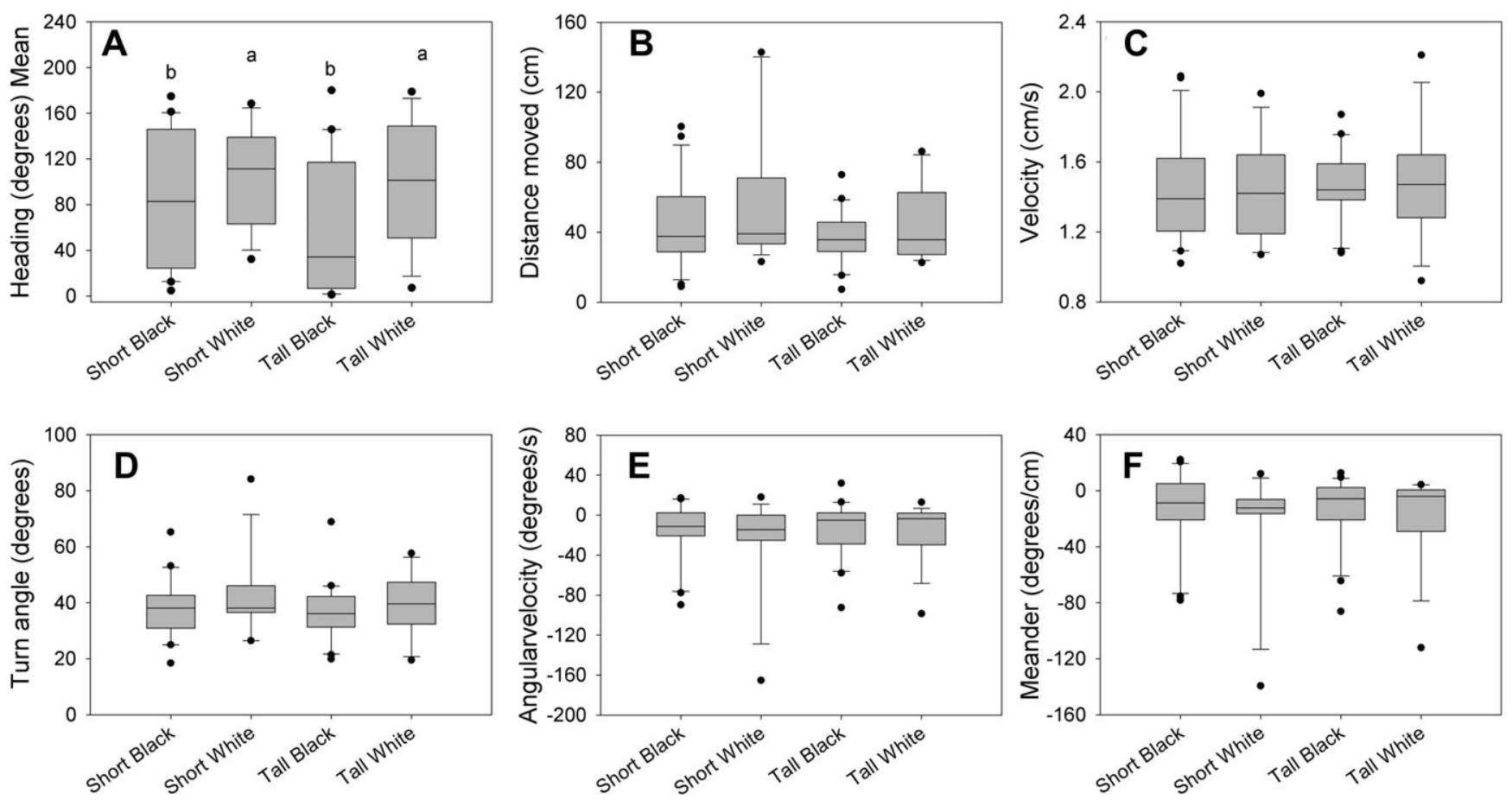

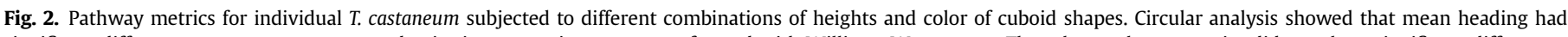

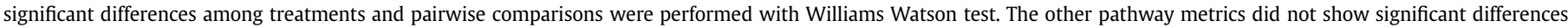
among the treatments when using GLM at a significance level of $P=0.05$.

shapes $(25.9 \pm 5.7)$ being greater than captures in traps with white shapes $(11.1 \pm 1.7)$ under low light intensity conditions (Man$\mathrm{n}$-Whitney test, $U=13.00, \mathrm{df}=14, P=0.05$ ). Under dark conditions, beetle captures in traps with black shapes $(11.5 \pm 1.6)$ and captures in traps with white shapes $(17.5 \pm 3.5)$ were not different from each other (paired $t$-test, $t=25.00, \mathrm{df}=14, P=0.50$ ). There was no significant difference in total number captured in both traps between low light intensity $(37.0 \pm 5.6)$ and dark conditions $(29.0 \pm 4.8)$ (paired $t$-test, $t=1.13, \mathrm{df}=14, P=0.28)$.
3.4. Experiment 4: does the use of black shape increase T. castaneum captures in pheromone and kairomone baited traps in a more complex landscape?

More beetles were captured in traps with black shapes than in traps with white shapes in the more complex landscapes, whether traps were placed in corners (paired $t$-test, $t=10.94$, df $=3$, $P=0.0016$ ) or along walls (paired $t$-test, $t=11.98, \mathrm{df}=3, P=0.036$ ) (Fig. 4). Although there was a trend for captures in traps with black

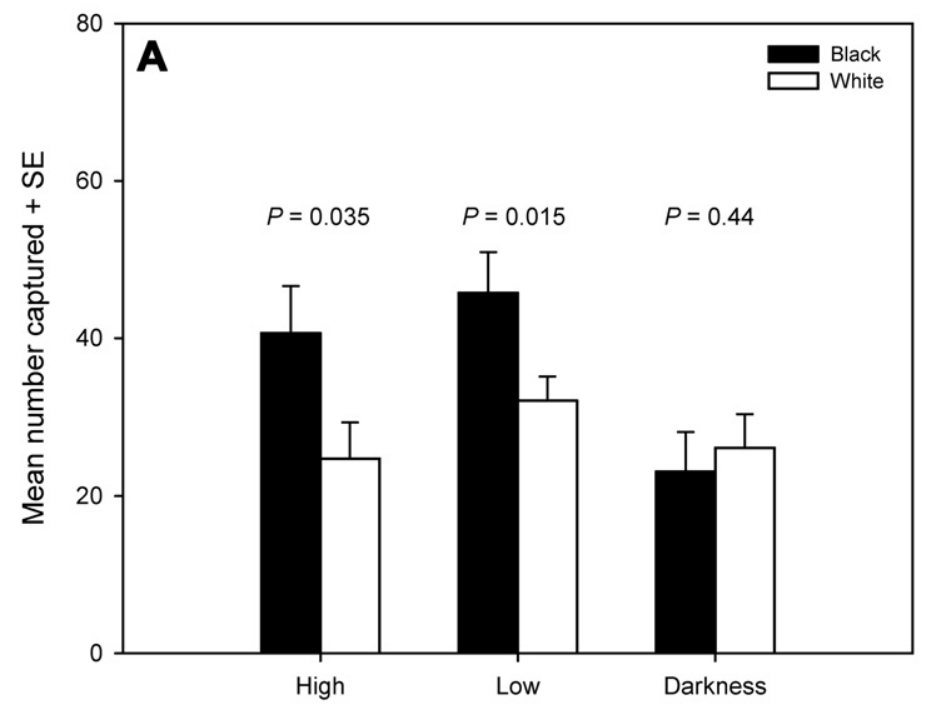

Light intensity
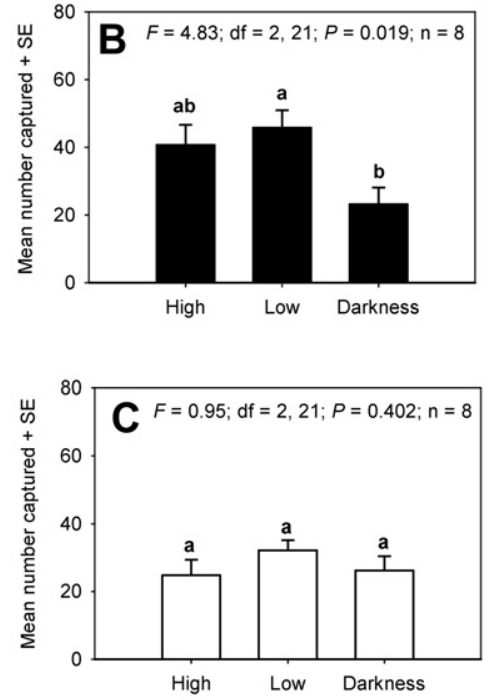

Light intensity

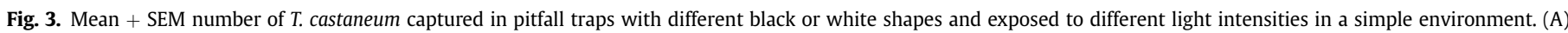

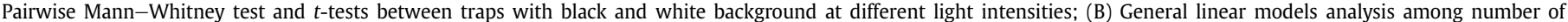

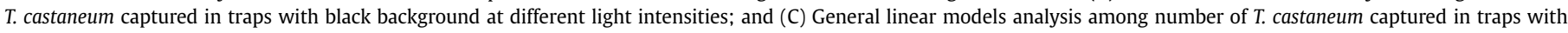
white background at different light intensities. 

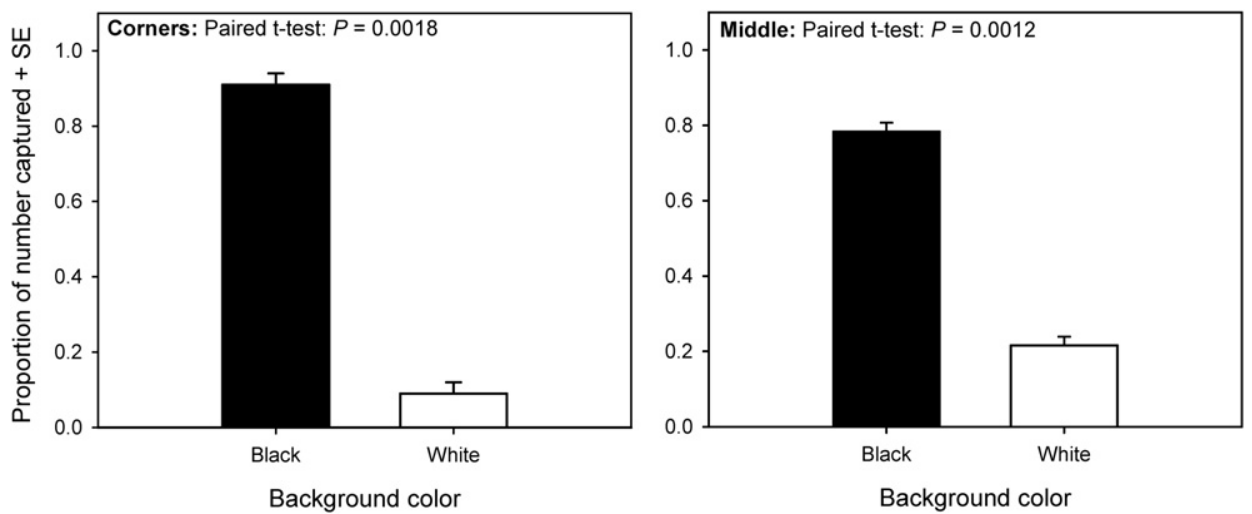

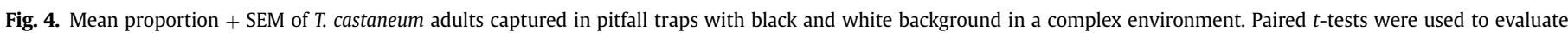
differences between traps located at the corners ("Corners" graph) and at the middle of two opposite walls ("Middle" graph).

shapes to be lower when placed along walls than in corners, this trend was not statistically significant (paired $t$-test, $t=3.02, \mathrm{df}=3$, $P=0.057)$.

\section{Discussion}

Additive effects of different sensory modes being used together as a tool for insect pest management has been shown for many species of insects (e.g., Strom and Goyer, 2001; Knight and Fisher, 2006; Reddy et al., 2009). However, the potential of combining sensory modes to increase trap captures is still relatively unknown for many stored-product pest species. Results of experiments described here show that $T$. castaneum adults are attracted to tall dark shapes and their presence behind traps can increase the number of adults captured. This combination approach might be useful in improving the effectiveness of these traps in food processing and storage facilities, although factors such as light intensity and contrast with color background may impact the level of increase. An advantage of this type of strategy is that it would be relatively inexpensive and easy to implement this combination; e.g., painting a black shape behind trap locations along a wall or placing traps near dark objects already present in facility.

Results of experiments 1 and 2 showed that against a white background, $T$. castaneum adults were more likely to respond to black shapes and that response increased with the height of the shape. That this response did not occur to white shapes when the background was black, suggests that beetles are not just responding to contrast with the background. Analysis of movement patterns in Experiment 2 also showed that $T$. castaneum adults appear to be attracted toward tall black shapes and not just arrested at them, since in the tall black shape treatments beetles reached the edge more quickly, moved in a non-random direction, and had a heading directed toward the shape. Reza and Parween (2006) showed that adult $T$. castaneum have a preference for black, but actually only measured arrestment on this color. Ours is the first study we are aware of showing that beetles actively orient toward the color black and also that the response is greater for taller black shapes. It is also interesting that fewer beetles attempted to fly when black pillars were present compared to white pillars against a white background, suggesting that absence of any color may increase flight initiation.

Placing dark tall shapes behind pheromone and kairomone baited traps increased beetle captures in both simple and complex landscapes (Experiments 3 and 4). The fact that increased response to traps with black color shapes was lost when experiments were performed in the dark also supports the hypothesis that it is visual cues that are enhancing trap response. Visual cues appear to attract more beetles to the vicinity of the traps and thus increasing their potential of being captured. Response to traps in front of dark shapes was also strong in experiment 4 where there were other competing darker shapes in the environment (e.g., table and wind tunnel) and beetles started off farther from the traps. The experiment with T. confusum supports the hypothesis that both of these species respond similarly to dark shapes, which could be important since both of these species are pests in the same types of facilities, and can co-occur in the same structures (Small, 2007).

Lighting in food processing facilities can be highly variable both temporally (e.g., lights on $24 \mathrm{~h}$ per day, lights off at night, lights on motion sensors) and spatially due to light fixture placement, window locations, and obstructions to light. For example, at one commercial food processing facility mean light intensity at 55 locations ranged from $1.4 \pm 0.5$ to $152.3 \pm 50.8$ lux, but most locations were below 50 lux (unpublished data). Insects have light thresholds necessary to perceive and discriminate different colors (Cammaerts and Cammaerts, 2009). In our experiments, T. castaneum could perceive and respond to the black shapes under low light intensity conditions ( $\sim 100$ lux). Interestingly, total trap captures were lowest under dark conditions, and while the mechanism needs further evaluation it suggests that either activity in general and/or attraction was also reduced under these conditions or that this represents the baseline level of response to olfactory cues alone. There was also a non-significant reduction in trap captures under high light intensity conditions, and our observations suggest that beetles were finding refugia and moving less under these conditions. Lighting patterns in food facilities could therefore have a significant impact on the pattern of trap captures and the potential benefits of adding dark shapes to traps. It will be necessary to evaluate impact of black backgrounds on beetle response to traps under a wider range of light intensities in order to determine if this behavioral response will increase trap captures in commercial facilities.

The reason for $T$. castaneum adult response to black shapes, either in terms of current fitness or evolutionary origin, is not known. Response to dark shapes might be related to current fitness benefits of finding covered areas for refugia that would be in shadow. These areas for refugia would have a greater probability of containing resources in terms of food, shelter, and finding mates. It may also be an evolutionary holdover and be related to ancestral response associated with exploiting trees. Sokoloff (1974) summarized the information available on natural habitats exploited by Tribolium spp. and concluded that this genus likely contained generalist feeders that exploited trees. Tribolium castaneum are occasionally found under the bark of trees and in acorns with longitudinally split seed coats (Subramanyam and Nelson, 1999; 
Bonneton, 2008). Some species of bark beetles (Coleoptera: Scolytidae) have been shown to respond to dark vertical shapes that represent the silhouette of trees (Campbell and Borden, 2006). The lesser grain borer Rhyzopertha dominica, a stored-product pest in the family Bostrichidae which contains primarily wood boring feeders, has been shown to respond to vertical silhouettes such as grain bins while flying (Mahroof et al., 2010). Tribolium castaneum is thought to be more of a scavenger or predator under tree bark than feeding directly on trees, but it may still have been selected to respond to cues that indicate trees. Tribolium castaneum has reduced color vision and enhanced UV and long wavelength light reception, suggesting cryptozoic origins (Jackowska et al., 2007). These combined factors may lead beetles to be responsive to these tall dark shapes since evolutionarily and currently they may indicate favorable habitats.

In conclusion, the results presented here demonstrated that T. castaneum and $T$. confusum adults respond strongly to tall and narrow black shapes and that these shapes can increase the capture of beetles in pheromone/kairomone baited traps when placed in front of them. This combination of olfactory and visual cues offers the potential to increase trap capture efficiency in monitoring programs. Further research will be needed to evaluate how much of an increase can be obtained in more complex environments such as those found inside food processing and storage facilities.

\section{Acknowledgments}

We thank Nickolas Kavallieratos for comments on an earlier version of this manuscript. This study was supported in part by USDA CSREES RAMP grant (Agreement No. 2005-51101-02358) and KAES contribution number 11-098-J. Mention of trade names or commercial products in this publication is solely for the purpose of providing specific information and does not imply recommendation or endorsement by the U. S. Department of Agriculture or Kansas State University.

\section{References}

Athanassiou, C.G., Kavallieratos, N.G., Mazomenos, B.E., 2004. Effect of trap type, trap color, trapping location and pheromone dispenser on captures of male Palpita unionalis (Hübner) (Lepidoptera: Pyralidae). Journal of Economic Entomology 97, 321-329.

Barak, A.V., Burkholder, W.E., Faustini, D.L., 1990. Factors affecting the design of traps for stored-products insects. Journal of Kansas Entomological Society 63, $466-485$.

Bonneton, F., 2008. The beetle by the name of Tribolium - typology and etymology of Tribolium castaneum Herbst, 1797. Insect Biochemistry and Molecular Biology 38, 377-379.

Burkholder, W.E., 1990. Practical use of pheromones and other attractants for stored-product insects. In: Ridgway, R.L., Silverstein, R.M., Inscoe, M.N. (Eds.), Behavior-modifying Chemicals for Insect Management: Applications of Pheromones and Other Attractants. Marcel Dekker, New York, pp. 497-516.
Cammaerts, M.-C., Cammaerts, D., 2009. Light thresholds for colour vision in workers of the ant Myrmica sabuleti (Hymenoptera: Formicidae). Belgian Journal of Zoology 139, 40-49.

Campbell, S.A., Borden, J.H., 2006. Integration of visual and olfactory cues of hosts and non-hosts by three bark beetles (Coleoptera: Scolytidae). Ecological Entomology 31, 437-449.

Campbell, J.F., Toews, M.D., Arthur, F.H., Arbogast, R.T., 2010. Long term monitoring of Tribolium castaneum in two flour mills: seasonal patterns and impact of fumigation. Journal of Economic Entomology 103, 991-1001.

Cilek, J.E., 2003. Attraction of colored plasticized corrugated boards to adult stable flies, Stomoxys calcitrans (Diptera: Muscidae). Florida Entomologist 86, 420-423.

Chambers, J., 1990. Overview on stored-product insect pheromones and food attractants. Journal of Kansas Entomological Society 63, 490-499.

Döring, T.F., Kirchner, S.M., Kühne, S., Saucke, H., 2004. Response of alate aphids to green targets on coloured backgrounds. Entomologia Experimentalis et Applicata $113,53-61$.

Finch, S., Collier, R.H., 2000. Host-plant selection by insects - a theory based on 'appropriate/inappropriate landings' by pest insects of cruciferous plants. Entomologia Experimentalis et Applicata 96, 91-102.

Hoback, W.W., Svatos, T.M., Spomer, S.M., Higley, L.G., 1999. Trap color and placement affects estimates of insect family-level abundance and diversity in a Nebraska salt marsh. Entomologia Experimentalis et Applicata 91, 393-402.

Jackowska, M., Bao, R., Liu, Z., McDonald, E.C., Cook, T.A., Friedrich, M., 2007. Genomic and gene regulatory signatures of cryptozoic adaptation: loss of blue sensitive photoreceptors through expansion of long wavelength-opsin expression in the red flour beetle Tribolium castaneum. Frontiers in Zoology 4, 1-11.

Knight, A.L., Fisher, J., 2006. Increased catch of Codling Moth (Lepidoptera: Tortricidae) in semiochemical-baited orange plastic delta-shaped traps. Environmental Entomology 35, 1597-1602.

Lanier, G.N., 1983. Integration of visual stimuli, host odorants and pheromones by bark beetles and weevils in locating and colonizing host trees. In: Ahmad, S. (Ed.), Herbivorous Insects: Host-seeking Behavior and Mechanisms. Academic Press, New York, pp. 161-171.

López-Guillén, G., Virgen, A., Rojas, J.C., 2009. Color preference of Anastrepha oblique (Diptera: Tephritidae). Revista Brasileira de Entomologia 53, 157-159.

Mahroof, R.M., Edde, P.A., Robertson, B., Puckette, J.A., Phillips, T.W., 2010. Dispersal of Rhyzopertha dominica (Coleoptera: Bostrichidae) in different habitats. Environmental Entomology 39, 930-938.

Mainali, B.P., Lim, U.T., 2010. Circular yellow sticky trap with black background enhances attraction of Frankliniella occidentalis (Pergande) (Thysanoptera: Thripidae). Applied Entomology and Zoology 45, 207-213.

Mullen, M.A., 1992. Development of a pheromone trap for monitoring Tribolium castaneum. Journal of Stored Product Research 28, 245-249.

Pinero, J.C., Jacome, I., Vargas, R., Prokopy, R.J., 2006. Response of female melon fly, Bractrocera cucurbitae, to host-associated visual and olfactory stimuli. Entomologia Experimentalis et Apllicata 121, 261-269.

Prokopy, R.J., Owens, E.D., 1983. Visual detection of plants by herbivorous insects. Annual Review of Entomology 28, 337-364.

Reddy, G.V.P., Cruz, Z.T., Guerrero, A., 2009. Development of an efficient pheromone-based trapping method for the banana root borer Cosmopolites sordidus. Journal of Chemical Ecology 35, 111-117.

Reza, A.M.S., Parween, S., 2006. Differential preference of colored surface in Tribolium castaneum (Herbst). Invertebrate Survival Journal 3, 84-88.

Small, G.J., 2007. A comparison between the impact of sulfuryl fluoride and methyl bromide fumigations on stored-product insect populations in UK flour mills. Journal of Stored Products Research 43, 410-416.

Sokoloff, A., 1974. The Biology of Tribolium, vol. Two. Oxford University Press, Oxford, UK.

Southwood, T.R.E., 1978. Ecological Methods. Chapman \& Hall, New York.

Strom, B.L., Goyer, R.A., 2001. Effect of silhouette color on trap catches of Dendroctonus frontalis (Coleoptera: Scolytidae). Annuals of the Entomological Society of America 94, 948-953.

Subramanyam, B.H., Nelson, J.J., 1999. Trapping indoors and out-lessons from the red flour beetle survey. The Sanitarian 3, 7-9.

Zar, J.H., 1999. Biostatistical Analysis, 4 ed. Prentice-Hall, Inc., New Jersey. 